\title{
Influence of hypoxia-related genetic polymorphisms on the prognosis of patients with metastatic gastric cancer treated with $\mathrm{EOF}$
}

\author{
WENBO TANG ${ }^{1,2^{*}}$, XIN LIU $^{1,2^{*}}$, LIXIN QIU ${ }^{1,2}$, XIAOYING ZHAO ${ }^{1,2}$, MINGZHU HUANG $^{1,2}$, \\ JILIANG YIN $^{1,2}$, JIN LI ${ }^{1,2}$, WEIJIAN GUO ${ }^{1,2}$, XIAODONG ZHU ${ }^{1,2}$ and ZHIYU CHEN ${ }^{1,2}$ \\ ${ }^{1}$ Department of Medical Oncology, Fudan University Shanghai Cancer Center; ${ }^{2}$ Department of Oncology, \\ Shanghai Medical College, Fudan University, Shanghai 200032, P.R. China
}

Received September 28, 2016; Accepted September 1, 2017

DOI: 10.3892/ol.2017.7414

\begin{abstract}
Tumor hypoxia is common in a number of solid tumor types including gastric cancer, and is associated with treatment resistance and poor prognosis. The present study aimed to investigate the function of hypoxia-associated genetic polymorphisms in predicting treatment response and survival in patients with metastatic gastric cancer (MGC) treated with EOF (oxaliplatin and 5-fluorouracil combined with epirubicin) as first-line chemotherapy. The present retrospective study enrolled 108 Chinese patients with MGC receiving EOF as first-line chemotherapy, and genotyped six single nucleotide polymorphisms (SNPs) in four hypoxia-associated genes [myoglobin (MB) rs7292 and rs7293, ATP Binding Cassette Subfamily G Member 2 rs2231142, MutL homolog 1 (MLH1) rs1800734 and rs9852810, and Poly(ADP-Ribose) Polymerase 1 rs1136410]. The results of the present study indicated that the CT/TT genotype of MB rs7292, as well as the GG genotype of MLH1 rs9852810, were independent favorable predictive factors of progression-free survival [PFS; MB rs7292: hazard ratio $(\mathrm{HR})=0.135$, 95\% confidence interval $(\mathrm{CI})=0.057-0.321, \mathrm{P}<0.001 ; \mathrm{MLH} 1$ rs9852810: $\mathrm{HR}=0.494,95 \% \mathrm{CI}=0.267-0.913, \mathrm{P}=0.024)$. Using a prognostic index based on the favorable SNPs for PFS (MB rs7292 CT/TT genotype, and MLH1 rs9852810 GG genotype), patients were classified into a low-risk group (involving one or two of the two SNPs) and a high-risk group (involving neither of the two SNPs), with a PFS of 180.0 and 117.0 days, respectively
\end{abstract}

Correspondence to: Dr Xiaodong Zhu or Dr Zhiyu Chen, Department of Oncology, Shanghai Medical College, Fudan University, 138 Yi Xue Yuan Road, Shanghai 200032, P.R. China

E-mail: xddr001@163.com

E-mail: chanhj75@aliyun.com

${ }^{*}$ Contributed equally

Key words: gastric cancer, epirubicin, oxaliplatin and 5-fluorouracil chemotherapy, single nucleotide polymorphism, survival, treatment response
$(\mathrm{P}=0.002)$. The results of the present study demonstrated that the CT/TT genotype of MB rs7292 and the GG genotype of MLH1 rs9852810 were independent favorable predictive factors of PFS in patients with MGC treated with EOF. Identification of those SNPs in blood samples may allow for the prediction of the short-term efficacy of first-line EOF treatment in patients with MGC.

\section{Introduction}

Notable progress has been achieved in previous years in the treatment of metastatic gastric cancer (MGC). For instance, trastuzumab combined with chemotherapy markedly prolongs overall survival (OS) in comparison with chemotherapy alone for patients with human epidermal growth factor receptor 2 (HER2)-positive MGC (1). However, HER2-positive disease accounts for only $7-34 \%$ of all gastric cancer (GC) (1); for patients with the HER2-negative disease, chemotherapy alone remains the standard treatment. EOF [oxaliplatin and 5-fluorouracil (5-FU) combined with epirubicin] is one of the classic first-line chemotherapeutic treatments for MGC. Nevertheless, $<50 \%$ of all patients respond to this treatment (2), driving the demand for predictive biomarkers in order to improve the selection of patients likely to respond to EOF therapy.

Tumor hypoxia develops in the majority of solid tumors due to the imbalance between the tumor growth and blood supply (3). In GC, previous studies have revealed that hypoxia may lead to drug resistance and stimulate the epithelial-mesenchymal transition (EMT) of GC cells $(4,5)$, and the presence of low levels of oxygenation in the tumor tissues of patients with $\mathrm{GC}$ has been associated with poor survival (6). Prior studies have additionally revealed that hypoxia-inducible factor (HIF)-1, the major transcription factor significantly activated by hypoxia, may confer hypoxia-induced drug resistance via the inhibition of drug-induced apoptosis, a decrease in intracellular drug accumulation and by prompting multidrug resistance (MDR) (7). Thus, the study of genes associated with tumor hypoxia may be of value in predicting the treatment response and prognosis of patients with MGC. 
Myoglobin (MB) is an oxygen-binding respiratory protein that has been identified in various non-muscle tissues (8). Previous studies have revealed that mRNA and/or protein levels of MB may be induced by hypoxia and were correlated with the expression of numerous hypoxia biomarkers, including HIF-1 $\alpha$, HIF-2 $\alpha$, and carbonic anhydrase IX (CAIX) in various types of cancer (9-13).

The ATP-binding cassette sub-family $G$ member 2 (ABCG2) promoter has been revealed to involve three hypoxia response elements (14). Under conditions of hypoxia, ABCG2 may be induced, thus the tumor cells would be provided with a survival advantage by reducing the accumulation of heme or porphyrin (15).

MutL homolog 1 (MLH1), a key DNA mismatch repair (MMR) gene, has been revealed to be specifically reduced in tumor cells and stem cells under hypoxia $(16,17)$, which eventually leads to genetic instability, tumor progression and resistance to chemotherapeutic agents including oxaliplatin, 5-FU, and irinotecan (18-21).

Poly(ADP-ribose) polymerase-1 (PARP-1) has been demonstrated to serve important functions in carcinogenesis (22). Previous studies have revealed that PARP-1 interacts with HIF- $1 \alpha$ and HIF-2 $\alpha(23,24)$, and that pre-exposure to hypoxia followed by oxidative stress may lead to the over activation of PARP-1 in human lung cancer cells (25).

In the present study, a retrospective analysis was conducted to evaluate the effect of 6 hypoxia-associated genetic polymorphisms (rs7292, rs7293, rs2231142, rs1800734, rs9852810, and rs1136410) on the treatment response and survival of Chinese patients with MGC receiving EOF chemotherapy.

\section{Materials and methods}

Patients. The present study retrospectively enrolled 108 Chinese patients with untreated MGC from May 2008 to June 2012 at the Fudan University Shanghai Cancer Center (Shanghai, China). All patients had pathologically diagnosed gastric adenocarcinoma with metastasis confirmed by magnetic resonance imaging or computed tomography, and were receiving the EOF treatment (intravenous epirubicin $50 \mathrm{mg} / \mathrm{m}^{2}$ combined with a $2 \mathrm{~h}$ intravenous infusion of oxaliplatin $130 \mathrm{mg} / \mathrm{m}^{2}$ on day 1 , followed by a $24 \mathrm{~h}$ continuous infusion of $5-F U 375-425 \mathrm{mg} / \mathrm{m}^{2} /$ day for 5 days) as first-line chemotherapy. The chemotherapy was repeated every 3 weeks until disease progression, intolerable toxicity, or withdrawal of consent. The response evaluation criteria in solid tumors (1.0) guidelines were used to evaluate tumor responses (26). Patients who achieved complete remission, partial remission, or stable disease were defined as 'controlled,' and patients with progressive disease were defined as 'uncontrolled.' Follow-up was conducted every 3-6 months, and OS was defined as the interval between the dates of the beginning of treatment and the first documentation of mortality from any cause, while progression-free survival (PFS) was defined as the interval between the dates of the beginning of treatment and the first documentation of disease progression or mortality from any cause.

The present study was approved by the Ethics Committee of Fudan University Shanghai Cancer Center (Shanghai, China) and was conducted in accordance with the Declaration of Helsinki. The blood samples obtained prior to treatment were acquired from the tissue bank of Fudan University Shanghai Cancer Center with written informed consent from the patients.

Genotyping. A total of six single nucleotide polymorphisms (SNP) in four hypoxia-related genes from the National Center for Biotechnology Information dbSNP database: MB (rs7292, rs7293), ABCG2 (rs2231142), MLH1 (rs1800734, rs9852810), and PARP-1 (rs1136410) were genotyped (Table I). Genomic DNA extraction and SNP genotyping were performed as described previously (27). Specifically, genomic DNA was extracted from the peripheral blood using the standard phenol-chloroform method. All probes and primers were designed by the Assay-on-Design service. The primer sequences were as follows: 1) rs7292, forward primer $\left(5^{\prime}-3^{\prime}\right)$ : ACGTTGGATGCTCATGATGCCCCTTCTTCT, reverse primer (5'-3'): ACGTTGGATGGAGGACTTAAAGAAGCA TGG, extension primer (5'-3'): tctgAAAGAAGCATGGTG CCAC. 2) rs7293, forward primer (5'-3'): ACGTTGGAT GGTTTGACAAGTTCAAGCACC, reverse primer $\left(5^{\prime}-3^{\prime}\right)$ : ACGTTGGATGTGGCACCATGCTTCTTTAAG, extension primer (5'-3'): GCTTCTTTAAGTCCTCAGA. 3) rs2231142, forward primer $\left(5^{\prime}-3^{\prime}\right)$ : ACGTTGGATGTGATGTTGTGA TGGGCACTC, reverse primer (5'-3'): ACGTTGGATGGT CATAGTTGTTGCAAGCCG, extension primer (5'-3'): ccc tCAAGCCGA AGAGCTGCTGAGAACT. 4) rs1800734, forward primer $\left(5^{\prime}-3^{\prime}\right)$ : ACGTTGGATGATCAATAGCTG CCGCTGAAG, reverse primer (5'-3'): ACGTTGGATGAG TGCCTCGTGCTCACGTTC, extension primer (5'-3'): gGC TCACGTTCTTCCTT. 5) rs9852810, forward primer (5'-3'): ACGTTGGATGTTTATGGAGCATCTACGGTG, reverse primer (5'-3'): ACGTTGGATGACTTTCCCTGCAGGGAT AAG, extension primer (5'-3'): atGGATAAGAGCATTAAA TGAGATAA. 6) rs1136410, forward primer (5'-3'): ACGTTG GATGTGAGCAGACTGTAGGCCAC, reverse primer $\left(5^{\prime}-3^{\prime}\right)$ : ACGTTGGATGGCTTTCTTTTGCTCCTCCAG, extension primer (5'-3'): ccttaCTTTTGCTCCTCCAGGCCAAGG.

All SNPs were genotyped with the TaqMan assay method using the ABI 7900 DNA Detection System (Applied Biosystems, Thermo Fisher Scientific, Inc., Waltham, MA, USA). The polymerase chain reaction (PCR) was performed using the TaqMan Universal PCR Master Mix reagent (Applied Biosystems; Thermo Fisher Scientific, Inc.) according to the manufacturer's protocol. PCR was performed at $95^{\circ} \mathrm{C}$ for $2 \mathrm{~min}$, followed by 45 cycles at $95^{\circ} \mathrm{C}$ for $30 \mathrm{sec}, 56^{\circ} \mathrm{C}$ for $30 \mathrm{sec}$ and $72^{\circ} \mathrm{C}$ for $1 \mathrm{~min}$, with a final incubation at $72^{\circ} \mathrm{C}$ for $5 \mathrm{~min}$; the extension reactions were conducted at $94^{\circ} \mathrm{C}$ for $30 \mathrm{sec}$ and then $94^{\circ} \mathrm{C}$ for $5 \mathrm{sec}$, followed by 5 cycles at $52^{\circ} \mathrm{C}$ for $5 \mathrm{sec}$ and at $80^{\circ} \mathrm{C}$ for $5 \mathrm{sec}$ for a total of 40 cycles and finally at $72^{\circ} \mathrm{C}$ for $3 \mathrm{~min}$.

Statistical analysis. Hardy-Weinberg equilibrium and pairwise linkage disequilibrium (LD), as well as allele and genotype distributions, were analyzed using SHEsis (http://analysis.bio-x.cn/myAnalysis.php) (28). The discrepancies in allelic and genotype frequency between the controlled and uncontrolled groups were analyzed using $\chi^{2}$ or Fisher's exact tests. OS and PFS were analyzed using the Kaplan-Meier method with the log-rank test using 
Table I. Summary of the six SNPs in the four hypoxia-related genes in the present study.

\begin{tabular}{|c|c|c|c|c|c|}
\hline Gene & SNP ID $^{\mathrm{a}}$ & Chromosome $^{\mathrm{b}}$ & Function & Allele & HW test P-value \\
\hline MB & rs7292 & ch.22:35610998 & Synonymous & $\mathrm{T}>\mathrm{C}$ & 0.912 \\
\hline MB & rs7293 & ch.22:35611028 & Synonymous & $\mathrm{G}>\mathrm{A}$ & 0.911 \\
\hline ABCG2 & rs2231142 & ch.4:88131171 & Missense & $\mathrm{C}>\mathrm{A}$ & 0.479 \\
\hline MLH1 & rs1800734 & ch.3:36993455 & 5'UTR & $\mathrm{G}>\mathrm{A}$ & 0.505 \\
\hline MLH1 & rs9852810 & ch. $3: 37027478$ & Intron variant & $\mathrm{G}>\mathrm{A}$ & 1.000 \\
\hline PARP-1 & rs1136410 & ch.1:226367601 & Missense & $\mathrm{T}>\mathrm{C}$ & 0.878 \\
\hline
\end{tabular}

${ }^{a}$ According to the dbSNP database; ${ }^{\text {T}}$ The SNP location based on the NCBI Human Genome Build 37.5. SNP, single nucleotide polymorphism; HW, Hardy-Weinberg; MB, myoglobin; ABCG2, ATP binding cassette subfamily G member 2; MLH1, MutL homolog 1; PARP-1, Poly(ADP-ribose) polymerase 1; UTR, untranslated region.

A

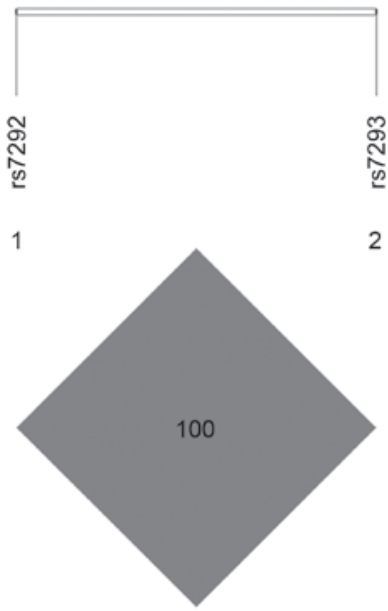

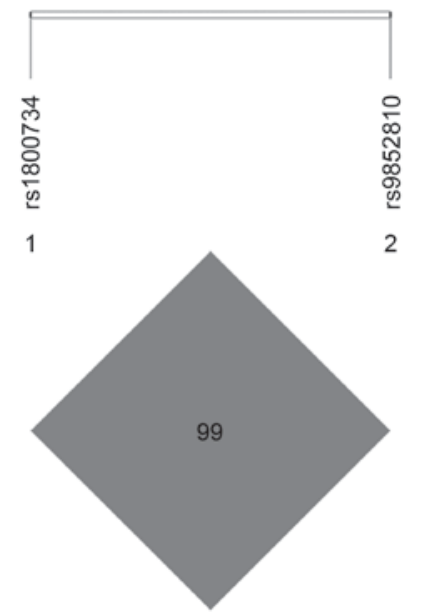

Figure 1. Linkage disequilibrium for the SNPs of MB and MLH1. (A) Linkage disequilibrium for MB SNPs rs7292 and rs7293; (B) Linkage disequilibrium for MLH1 SNPs rs1800734 and rs9852810. SNP, single nucleotide polymorphism; MB, myoglobin; MLH1, MutL homolog 1.

SPSS software (version 19.0; IBM Corp., Armonk, NY, USA). Risk factors with $\mathrm{P}<0.1$ were further analyzed using multivariate Cox regression models to assess the influence of genotypes on treatment-association survival. The software Testing Haplotype Effects in Association Studies (version 3.1) was used to perform haplotype analysis (29). A two-tailed $\mathrm{P}<0.05$ was considered to indicate a statistically significant difference.

\section{Results}

Study population and SNPS. The study population included 108 Chinese patients with MGC receiving EOF chemotherapy. Baseline demographic and clinical data were described in a previous study (30). In brief, $41.0 \%$ (44/108) of the patients were female, and the age of the patients ranged from 23-74 years. Within the study population, 95.4\% (103/108) of patients had an Eastern Cooperative Oncology Group (ECOG) score (31) of $0-1$. All the patients had at least one unresectable lesion including lung metastasis, liver metastasis, ascites, pleural effusion or retroperitoneal lymph node invasion. According to the best treatment response, $89(82.4 \%)$ patients were regarded as controlled and $19(17.6 \%)$ patients were uncontrolled. There was no difference between the controlled and uncontrolled patients in terms of sex, age, ECOG score, tumor differentiation, synchronous metastasis status and number of lesions. The median follow-up duration was 28.4 (range, 7.7-69.0) months.

Table I presents the 6 SNPs analyzed in the present study. Each of the SNPs was in Hardy-Weinberg equilibrium. Patients for whom data was lacking for certain SNPs (for instance, 1/108 patients for rs2231142) were excluded from the corresponding analysis.

LD analysis. Pairwise LD analysis revealed strong $\mathrm{LD}$ in MB SNPs rs7292 and rs7293 ( $D^{\prime}=1.000, r^{2}=1.000$; Fig. 1A), as well as MLH1 SNPs rs1800734 and rs9852810 ( $\mathrm{D}^{\prime}=1.000$, $r^{2}=0.084$; Fig. 1B). Since rs7292 and rs7293 were in complete LD ( $\left.\mathrm{D}^{\prime}=1.000, \mathrm{r}^{2}=1.000\right)$, only rs7292 was included in the subsequent analysis to avoid unnecessary duplication.

Association of alleles and genotype frequency with disease control. Differences between the four SNPs analyzed in the present study and disease control are presented in Table II. 


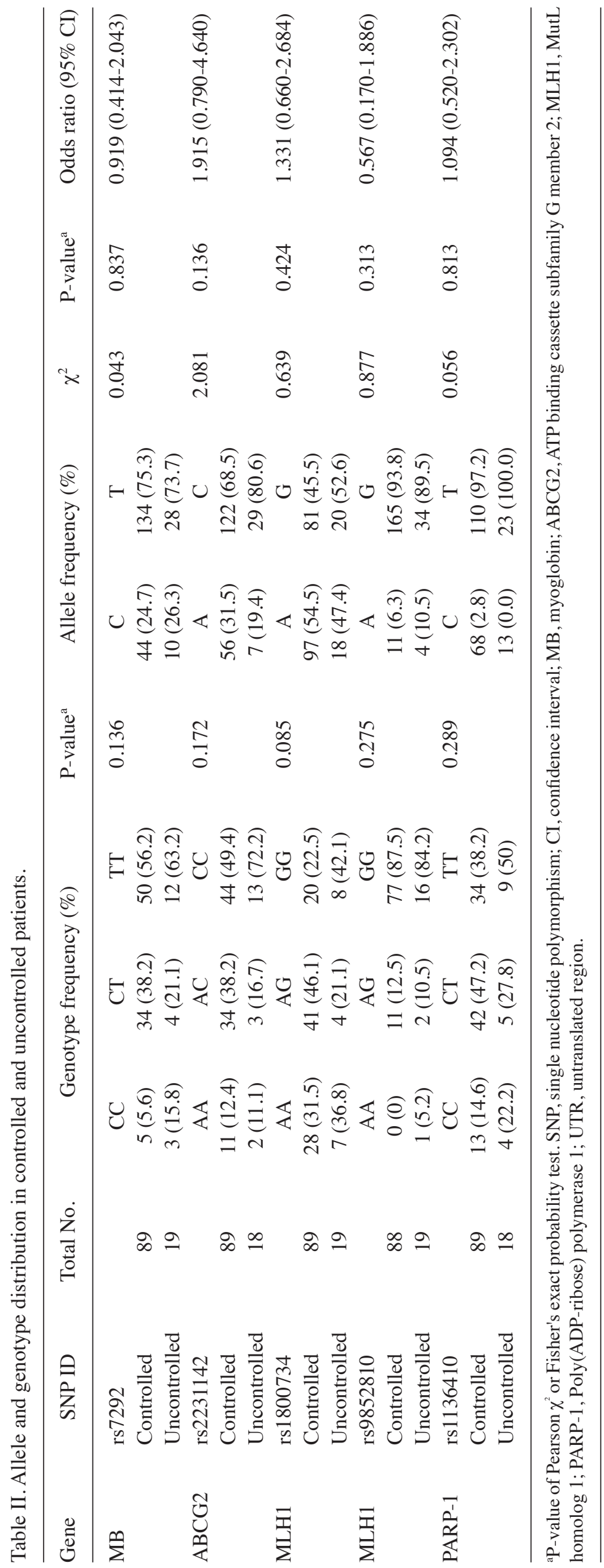


Table III. PFS and OS analysis with the Kaplan-Meier method and log-rank test.

\begin{tabular}{llccccccc}
\hline $\begin{array}{l}\text { Clinical } \\
\text { characteristic }\end{array}$ & $\begin{array}{l}\text { Clinical } \\
\text { factors }\end{array}$ & Patients (n) & $\begin{array}{c}\text { Median } \\
\text { PFS (days) }\end{array}$ & $95 \%$ CI & P-value $^{\mathrm{a}}$ & $\begin{array}{c}\text { Median } \\
\text { OS (days) }\end{array}$ & $95 \%$ CI & P-value $^{\mathrm{a}}$ \\
\hline MB rs7292 & CC & 8 & 71.0 & $50.2-91.8$ & 0.001 & 244.0 & $132.4-355.6$ & 0.245 \\
& CT+TT & 100 & 179.0 & $162.7-191.1$ & & 465.0 & $302.8-627.2$ & \\
ABCG2 rs2231142 & AA & 13 & 182.0 & $159.2-204.7$ & 0.705 & 297.0 & $173.2-420.8$ & 0.278 \\
& AC+CC & 94 & 173.0 & $152.3-193.7$ & & 465.0 & $292.0-638.0$ & \\
MLH1 rs1800734 & AA & 35 & 159.0 & $112.0-206.0$ & 0.897 & 367.0 & $50.3-683.7$ & 0.590 \\
& AG+GG & 73 & 179.0 & $158.5-199.5$ & & 465.0 & $309.3-620.7$ & \\
MLH1 rs9852810 & AA+AG & 14 & 147.0 & $51.7-242.3$ & 0.040 & 241.0 & $100.0-382.1$ & 0.287 \\
& GG & 93 & 178.0 & $155.8-200.2$ & & 465.0 & $207.7-622.3$ & \\
PARP-1 rs1136410 & CC & 17 & 202.0 & $158.0-246.0$ & 0.528 & 1378.0 & b & 0.156 \\
& CT+TT & 90 & 169.0 & $148.1-189.9$ & & 403.0 & $252.4-533.6$ & \\
\hline
\end{tabular}

aP-value of the log-rank test. ${ }^{\text {b } 95 \% ~ C I ~ c a n n o t ~ b e ~ c a l c u l a t e d ~ d u e ~ t o ~ t h e ~ f a c t ~ t h a t ~} 10$ out of 17 patients in the subgroup are censored. OS, overall survival time; PFS, progression-free survival; MB, myoglobin; ABCG2, ATP binding cassette subfamily G member 2; MLH1, MutL homolog 1; PARP-1, Poly(ADP-ribose) polymerase 1; UTR, untranslated region.

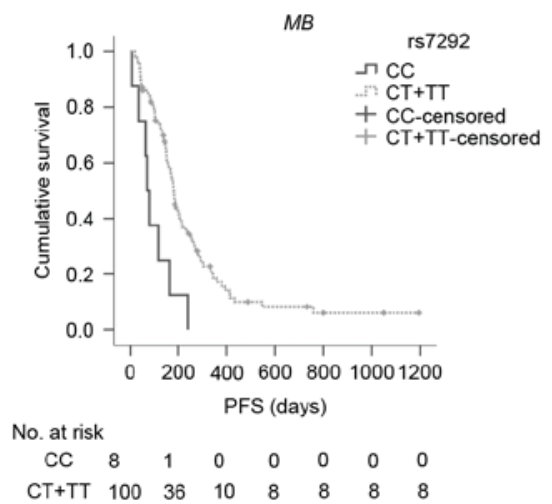

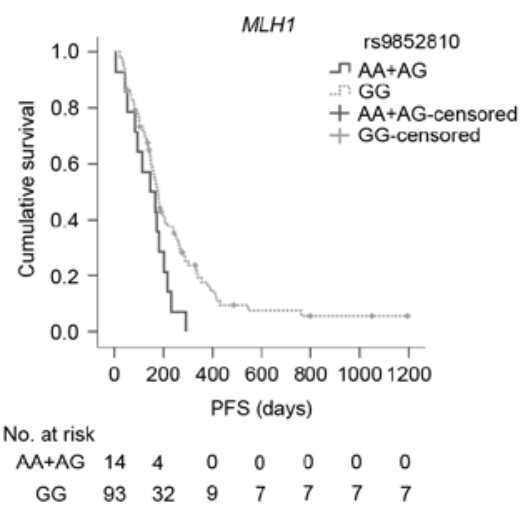

C

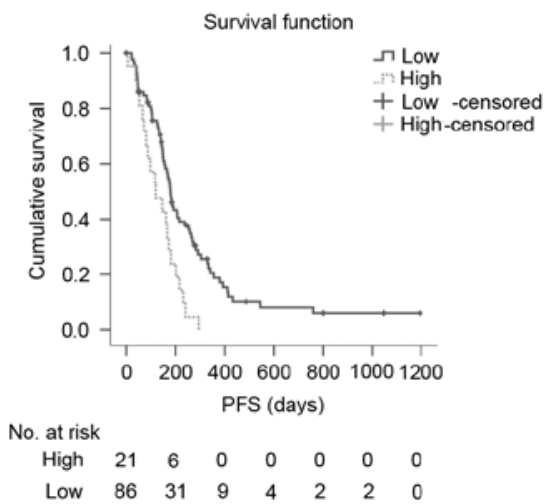

Figure 2. PFS curves for MB rs7292, MLH1 rs9852810, and the prognostic index among subgroups. (A) PFS curves of rs7292 among subgroups. Median PFS time for CC carriers (solid line) and CT/TT carriers (dotted line) were 71.0 days (95\% CI: 50.2-91.8) and 179.0 days (95\% CI: 162.7-191.1), respectively $(\mathrm{P}=0.001)$; (B) PFS curves of rs9852810 among subgroups. Median overall survival time for AA/AG carriers (solid line), GG carriers (dotted line) were 147.0 days (95\% CI: 51.7-242.3) and 178.0 days (95\% CI: 155.8-200.2), respectively (P=0.040); (C) PFS curves of prognostic index among subgroups. The median PFS time for low-risk (0 risk, solid line), and high-risk (1 or 2 risks, dotted line) were 180.0 days (95\% CI: 161.5-198.5) and 117.0 days (95\% CI: 40.7-193.3) respectively $(\mathrm{P}=0.002)$. The high-risk group had a 2.2-fold increased risk of progression compared with the low-risk group $(\mathrm{HR}=2.223,95 \% \mathrm{CI}=1.335-3.704$; $\mathrm{P}=0.002)$. PFS, progression-free survival; $\mathrm{CI}$, confidence interval; HR, hazard ratio; MB, myoglobin; MLH1, MutL homolog 1.

No allele or genotype exhibited a significant association with treatment response.

Survival analysis. In addition to pathological grade and number of lesions, as reported in a previous study (27), the genotypes of MB rs7292 and MLH1 rs9852810 were revealed to be significantly associated with PFS (Table III). T carriers of MB rs7292 had significantly more favorable PFS compared with CC carriers (median PFS: 179.0 and 71.0 days, respectively $\mathrm{P}=0.001$; Fig. 2A). Similarly, GG carriers of MLH1 rs9852810 had significantly longer PFS in comparison to A carriers (median PFS: 178.0 and 147.0 days, respectively; $\mathrm{P}=0.040$; Fig. 2B). In the Cox regression models, the T allele of MB rs7292 and the GG genotype of MLH1 rs9852810 were associated with decreased risks of disease progression [rs7292: hazard ratio $(\mathrm{HR})=0.135$, $95 \%$ confidence interval $(\mathrm{CI})=0.057-0.321 ; \mathrm{P}<0.001 ;$ rs9852810:
$\mathrm{HR}=0.494,95 \% \mathrm{CI}=0.267-0.913 ; \mathrm{P}=0.024$, respectively], indicating that, along with pathological grade $(\mathrm{P}=0.005)$ and number of lesions ( $\mathrm{P}=0.020), \mathrm{MB}$ rs7292 and MLH1 rs9852810 were independent predictive factors of PFS for patients with MGC receiving EOF chemotherapy (Table IV). However, none of the SNPs were revealed to have an association with OS using Kaplan-Meier analysis (Table III).

PFS analysis based on the prognostic index. According to the favorable SNPs for PFS (MB rs7292 CT/TT genotype and MLH1 rs9852810 GG genotype), patients were classified into a low-risk group (involving one or two of the two SNPs) and a high-risk group (involving neither of the two SNPs). Patients from the low-risk group revealed a significantly longer PFS than patients from the high-risk group (median PFS: 180.0 and 117.0 days, respectively; $\mathrm{P}=0.002$; Fig. 2C). In comparison 
Table IV. Multivariate analysis of prognostic factors of PFS following EOF treatment.

\begin{tabular}{|c|c|c|c|}
\hline \multirow{2}{*}{$\begin{array}{l}\text { Clinical } \\
\text { characteristic }\end{array}$} & \multicolumn{3}{|c|}{ PFS } \\
\hline & Hazard ratio & $95 \% \mathrm{CI}$ & P-value ${ }^{a}$ \\
\hline \multicolumn{4}{|l|}{ Pathological grade } \\
\hline $\begin{array}{l}\text { Low and } \\
\text { undifferentiated }\end{array}$ & 1 & & 0.005 \\
\hline Moderate and high & 0.252 & $0.097-0.657$ & \\
\hline Unclassified & 0.633 & $0.382-1.046$ & \\
\hline \multicolumn{4}{|l|}{ No. of lesions } \\
\hline 1 & 1 & & 0.020 \\
\hline 2 & 0.159 & $0.030-0.854$ & \\
\hline$\geq 3$ & 0.952 & $0.300-3.025$ & \\
\hline \multicolumn{4}{|c|}{ Synchronous metastasis } \\
\hline Presence & 1 & & 0.628 \\
\hline Absence & 1.184 & $0.597-2.348$ & \\
\hline \multicolumn{4}{|l|}{ MB rs7292 } \\
\hline $\mathrm{CC}$ & 1 & & $<0.001$ \\
\hline $\mathrm{CT}+\mathrm{TT}$ & 0.135 & $0.057-0.321$ & \\
\hline \multicolumn{4}{|l|}{ MLH1 rs9852810 } \\
\hline $\mathrm{AA}+\mathrm{AG}$ & 1 & & 0.024 \\
\hline GG & 0.494 & $0.267-0.913$ & \\
\hline
\end{tabular}

aP-value is evaluated in patients without an unclassified pathological stage. PFS, progression-free survival; CI, confidence interval; MB, myoglobin; MLH1, MutL homolog 1.

with the low-risk group, the high-risk group demonstrated a 2.2-fold increased risk of disease progression $(\mathrm{HR}=2.223$, $95 \% \mathrm{CI}=1.335-3.704 ; \mathrm{P}=0.002)$.

Haplotype analysis. Haplotype analysis was performed in SNPs that were in strong LD. The analysis demonstrated that the GA haplotype of MLH1 rs1800734/rs9852810 was associated with unfavorable PFS $(\mathrm{HR}=1.84,95 \% \mathrm{CI}=1.07-3.16$; $\mathrm{P}=0.027$ ), while no haplotype of MLH1 rs1800734/rs9852810 was associated with OS (Table V). The haplotypes of MB had no significant influence on survival (Table VI).

\section{Discussion}

Hypoxia is common in numerous types of solid tumor, including GC, and is considered to be an important factor associated with aggressive phenotypes and treatment resistance of tumors $(6,32)$. Several studies have investigated the influence of hypoxia on the chemosensitivity, metastasis, and prognosis of GC. Liu et al (4) demonstrated that GC cells developed resistance to 5-FU, vincristine, cisplatin, etoposide, and adriamycin under conditions of hypoxia. Matsuoka et al (5) demonstrated that hypoxia may stimulate the EMT process of GC cells. In addition, Osinsky et al (6) revealed that low levels of oxygenation in tumor tissues were associated with poor survival in patients with GC.
The present study explored the relationship between polymorphisms of genes associated with tumor hypoxia and the clinical outcome of patients with MGC treated with $\mathrm{EOF}$, and to the best of our knowledge, revealed for the first time that the CT/TT genotype of MB rs7292 and the GG genotype of MLH1 rs9852810 were independent favorable predictive factors of PFS for patients with MGC treated with the EOF regimen. Since PFS more directly reflects the therapeutic effect of first-line chemotherapy, MB rs7292 and MLH1 rs9852810 may serve as biomarkers that predict the short-term efficacy of the first-line EOF regimen in patients with MGC.

The MB rs7292 CT/TT genotype was demonstrated to be an independent favorable predictor of PFS for patients with MGC treated with EOF chemotherapy, while MB rs7292 CT/TT carriers appeared to possess a tendency toward an improved OS compared with CC carriers (for CT/TT and CC genotypes, median OS: 465.0 and 244.0 days, respectively; $\mathrm{P}=0.245$ ). Although the difference was not statistically significant, considering the markedly separative tendency of OS curves and the limited sample size of the present study, there may still be a link between the CT/TT genotype and survival. With respect to the prognostic function of MB rs7293, since MB rs7292 and rs7293 were in complete $L D\left(D^{\prime}=1.000, r^{2}=1.000\right)$, the results of the analysis of the rs7293 GG genotype were identical to those of the rs7292 CC genotype (data not shown).

The function of MB in GC has not previously been reported, and the function of $\mathrm{MB}$ in tumors varies and remains controversial. For example, one previous study demonstrated that $71 \%$ of 1,027 breast cancer cases demonstrated MB expression, with a significant correlation with hypoxia markers (HIF-2 $\alpha$, CAIX) and an improved prognosis (9). Furthermore, another previous study revealed that MB was induced in breast cancer cells by prolonged hypoxia, partially by HIF-1/2-dependent transactivation, and additionally revealed that MB may damage mitochondria in hypoxic cancer cells, indicating the tumor-suppressive potential of $\mathrm{MB}$ in breast cancer (10). However, the function of $\mathrm{MB}$ in non-small-cell lung cancer is controversial. For instance, Galluzzo et al (33) demonstrated that overexpression of MB in non-small-cell lung cancer cells suppressed the hypoxia response, inhibited tumor growth, reduced vessel density, promoted tumor differentiation and suppressed tumor metastasis. On the other hand, Oleksiewicz et al (13) reported that patients with non-small-cell lung cancer with low MB levels had a longer survival duration than patients with high $\mathrm{MB}$ expression. Hypoxia may also induce the expression of $\mathrm{MB}$ in prostate cancer (11) and renal cell carcinoma (12). However, to date, no studies have reported the polymorphisms of $\mathrm{MB}$ in malignant tumors, and the function of $\mathrm{MB}$ in gastric cancer has not been previously demonstrated. Further research is required to elucidate whether the change of rs7292 may affect the expression of MB, and whether and how MB may influence the efficacy of the EOF treatment in patients with GC.

The link between MLH1 rs1800734 and rs9852810 and $\mathrm{GC}$ was also reported in the present study. In addition to the prolonged PFS of patients with MGC carrying the GG genotype of MLH1 rs9852810, MLH1 rs9852810 GG carriers were revealed to possess an improved OS 
Table V. Analyses of associations between haplotypes of MLH1 and PFS/OS.

\begin{tabular}{|c|c|c|c|c|c|c|c|}
\hline \multirow[b]{2}{*}{ Haplotypes } & \multirow[b]{2}{*}{ Haplotype frequencies (\%) } & \multicolumn{3}{|c|}{ PFS } & \multicolumn{3}{|c|}{ OS } \\
\hline & & HR & $95 \%$ CI & P-value & HR & $95 \%$ CI & P-value \\
\hline $\mathrm{AG}^{\mathrm{a}}$ & 51.00 & & & & & & \\
\hline $\mathrm{GA}^{\mathrm{a}}$ & 6.73 & 1.84 & $1.07-3.16$ & 0.027 & 1.50 & $0.75-3.00$ & 0.253 \\
\hline $\mathrm{GG}^{\mathrm{a}}$ & 42.31 & 0.95 & $0.70-1.27$ & 0.712 & 0.87 & $0.58-1.30$ & 0.490 \\
\hline
\end{tabular}

${ }^{a}$ Haplotype was composed in the order of rs1800734 then rs9852810; AG was the most common haplotype. PFS, progression-free survival; OS, overall survival; CI, confidence interval; HR, hazard ratio; MLH1, MutL homolog 1.

Table VI. Analyses of associations between haplotypes of MB and PFS/OS.

\begin{tabular}{|c|c|c|c|c|c|c|c|}
\hline \multirow[b]{2}{*}{ Haplotypes } & \multirow[b]{2}{*}{ Haplotype frequencies $(\%)$} & \multicolumn{3}{|c|}{ PFS } & \multicolumn{3}{|c|}{ OS } \\
\hline & & HR & $95 \% \mathrm{CI}$ & P-value & HR & $95 \%$ CI & $\mathrm{P}$-value \\
\hline $\mathrm{TA}^{\mathrm{a}}$ & 75.00 & & & & & & \\
\hline $\mathrm{CG}^{\mathrm{a}}$ & 25.00 & 1.09 & $0.79-1.51$ & 0.60 & 1.20 & $0.78-1.84$ & 0.41 \\
\hline
\end{tabular}

compared with A carriers (for GG and AA/AG genotypes, median OS: 465.0 and 241.0 days, respectively; $\mathrm{P}=0.287$ ). The results demonstrating that OS difference did not reach statistical significance may be attributed to the relatively small sample size and the therapy following first-line chemotherapy. Additionally, haplotype analysis revealed that the GA haplotype of MLH1 rs1800734/rs9852810 was associated with poorer PFS compared with the common haplotype AG; the reason may be that the GG genotype of rs9852810 had a significantly favorable PFS.

As a key DNA MMR gene, the expression of MLH1 was demonstrated to be reduced under hypoxia as a result of transcriptional, translational, or methylation modification $(18,34,35)$. In addition, several studies have revealed that methylation of the MLH1 gene promoter was associated with the stages of carcinogenesis and progression as well as oxaliplatin resistance in GC (36-38).

With respect to the influence of the polymorphisms of MLH1 on cancer, Langeberg et al (39) reported that the rs9852810 A allele was associated with a higher risk of prostate cancer incidence and recurrence, as well as being associated with more aggressive types of prostate cancer. Furthermore, rs1800734 was located in the CpG island of MLH1, and the A allele of rs1800734 was significantly associated with MLH1 methylation and high-degree microsatellite instability in colorectal cancer (40-42). In GC, Zhu et al (43) demonstrated that the G allele of MLH1 rs1800734 was associated with a decreased risk of GC. However, the present study demonstrated no significant association between rs1800734 and the clinical outcome of patients with MGC who received EOF chemotherapy, a result that may be explained by the controversy surrounding the influence of microsatellite instability on GC $(44,45)$. Since the influence of the two polymorphisms on the survival and treatment response of patients with MGC has, to the best of our knowledge, never previously been reported, and additionally whether the two SNPs may alter the function of MLH1 and subsequently influence GC progression is unknown, additional work is required to validate the predictive value of those polymorphisms for GC.

When the patients were classified into a low-risk group and a high-risk group according to the number of favorable SNPs for PFS (MB rs7292 CC genotype and MLH1 rs9852810 A allele) they possessed, the high-risk group was revealed to possess a significantly shorter PFS; thus, this type of classification may assist in a more comprehensive identification of patients with poor prognosis.

To conclude, to the best of our knowledge, the present study revealed for the first time, that in Chinese patients with MGC treated with the EOF regimen as first-line chemotherapy, the CT/TT genotype of MB rs7292 and the GG genotype of MLH1 rs9852810 were independent favorable predictive factors of PFS, and that these may serve as biomarkers to predict the short-term efficacy of the first-line EOF regimen in patients with MGC. Since all the SNPs of the four hypoxia-associated genes investigated in the present study have seldom been reported in GC, the results of the present study will require confirmation with larger cohort studies and further functional investigations.

\section{Acknowledgements}

The present study was supported by the Natural Science Foundation of Shanghai, Shanghai, China (grant 
no. 13ZR1408200) and the Foundation of the Shanghai Municipal Science and Technology Commission, Shanghai, China (grant no. 134119a8600).

\section{References}

1. Bang YJ, Van Cutsem E, Feyereislova A, Chung HC, Shen L, Sawaki A, Lordick F, Ohtsu A, Omuro Y, Satoh T, et al: Trastuzumab in combination with chemotherapy versus chemotherapy alone for treatment of HER2-positive advanced gastric or gastro-oesophageal junction cancer (ToGA): A phase 3 , open-label, randomised controlled trial. Lancet 376: 687-697, 2010.

2. Van Cutsem E, Moiseyenko VM, Tjulandin S, Majlis A, Constenla M, Boni C, Rodrigues A, Fodor M, Chao Y, Voznyi E, et al: Phase III study of docetaxel and cisplatin plus fluorouracil compared with cisplatin and fluorouracil as first-line therapy for advanced gastric cancer: A report of the V325 study group. J Clin Oncol 24: 4991-4997, 2006.

3. Balkwill FR, Capasso M and Hagemann T: The tumor microenvironment at a glance. J Cell Sci 125: 5591-5596, 2012.

4. Liu L, Ning X, Sun L, Zhang H, Shi Y, Guo C, Han S, Liu J, Sun S, Han Z, Wu K and Fan D: Hypoxia-inducible factor-1 alpha contributes to hypoxia-induced chemoresistance in gastric cancer. Cancer Sci 99: 121-128, 2008.

5. Matsuoka J, Yashiro M, Doi Y, Fuyuhiro Y, Kato Y, Shinto O, Noda S, Kashiwagi S, Aomatsu N, Hirakawa T, et al: Hypoxia stimulates the EMT of gastric cancer cells through autocrineTGFbeta signaling. PLoS One 8: e62310, 2013.

6. Osinsky S, Bubnovskaya L, Ganusevich I, et al: Hypoxia, tumourassociated macrophages, microvessel density, VEGF and matrix metalloproteinases in human gastric cancer: interaction and impact on survival. Clinical Translational Oncol 13: 133-138, 2011.

7. Röhwer N, Dame C, Haugstetter A, Wiedenmann B, Detjen K, Schmitt CA and Cramer T: Hypoxia-inducible factor lalpha determines gastric cancer chemosensitivity via modulation of p53 and NF-kappaB. PLoS One 5: e12038, 2010.

8. Fraser J, de Mello LV, Ward D, Rees HH, Williams DR, Fang Y, Brass A, Gracey AY and Cossins AR: Hypoxia-inducible myoglobin expression in nonmuscle tissues. Proc Natl Acad Sci USA 103: 2977-2981, 2006.

9. Kristiansen G, Rose M, Geisler C, Fritzsche FR, Gerhardt J, Lüke C, Ladhoff AM, Knüchel R, Dietel M, Moch H, et al: Endogenous myoglobin in human breast cancer is a hallmark of luminal cancer phenotype. Br J Cancer 102: 1736-1745, 2010.

10. Kristiansen G, Hu J, Wichmann D, Stiehl DP, Rose M, Gerhardt J, Bohnert A, Ten Haaf A, Moch H, Raleigh J, et al: Endogenous myoglobin in breast cancer is hypoxia-inducible by alternative transcription and functions to impair mitochondrial activity: A role in tumor suppression? J Biol Chem 286: 43417-43428, 2011.

11. Meller S, Bicker A, Montani M, Ikenberg K, Rostamzadeh B, Sailer V, Wild P, Dietrich D, Uhl B, Sulser T, et al: Myoglobin expression in prostate cancer is correlated to androgen receptor expression and markers of tumor hypoxia. Virchows Arch 465: 419-427, 2014

12. Behnes CL, Bedke J, Schneider S, Küffer S, Strauss A, Bremmer F, Ströbel P and Radzun HJ: Myoglobin expression in renal cell carcinoma is regulated by hypoxia. Exp Mol Pathol 95 307-312, 2013

13. Oleksiewicz U, Daskoulidou N, Liloglou T, Tasopoulou K, Bryan J, Gosney JR, Field JK and Xinarianos G: Neuroglobin and myoglobin in non-small cell lung cancer: Expression, regulation and prognosis. Lung Cancer 74: 411-418, 2011.

14. Krishnamurthy $\mathrm{P}$ and Schuetz JD: The ABC transporter Abcg2/Bcrp: Role in hypoxia mediated survival. Biometals 18 349-358, 2005.

15. Krishnamurthy P, Ross DD, Nakanishi T, Bailey-Dell K, Zhou S, Mercer KE, Sarkadi B, Sorrentino BP and Schuetz JD: The stem cell marker Bcrp/ABCG2 enhances hypoxic cell survival through interactions with heme. J Biol Chem 279: 24218-24225, 2004.

16. Mihaylova VT, Bindra RS, Yuan J, Campisi D, Narayanan L, Jensen R Giordano F, Johnson RS, Rockwell S and Glazer PM: Decreased expression of the DNA mismatch repair gene Mlh1 under hypoxic stress in mammalian cells. Mol Cell Biol 23: 3265-3273, 2003.

17. Rodríguez-Jiménez FJ, Moreno-Manzano V, Lucas-Dominguez R and Sánchez-Puelles JM: Hypoxia causes downregulation of mismatch repair system and genomic instability in stem cells. Stem Cells 26: 2052-2062, 2008
18. Nakamura $\mathrm{H}$, Tanimoto $\mathrm{K}$, Hiyama $\mathrm{K}$, Yunokawa $\mathrm{M}$, Kawamoto T, Kato Y, Yoshiga K, Poellinger L, Hiyama E and Nishiyama M: Human mismatch repair gene, MLH1, is transcriptionally repressed by the hypoxia-inducible transcription factors, DEC1 and DEC2. Oncogene 27: 4200-4209, 2008.

19. Li Y, Yang Y, Lu Y, Herman JG, Brock MV, Zhao P and Guo M: Predictive value of CHFR and MLH1 methylation in human gastric cancer. Gastric Cancer 18: 280-287, 2015.

20. Meyers M, Wagner MW, Hwang HS, Kinsella TJ and Boothman DA: Role of the hMLH1 DNA mismatch repair protein in fluoropyrimidine-mediated cell death and cell cycle responses. Cancer Res 61: 5193-5201, 2001.

21. Tentori L, Leonetti C, Muzi A, Dorio AS, Porru M, Dolci S, Campolo F, Vernole P, Lacal PM, Praz F and Graziani G: Influence of MLH1 on colon cancer sensitivity to poly(ADP-ribose) polymerase inhibitor combined with irinotecan. Int J Oncol 43: 210-218, 2013.

22. Vyas S and Chang P: New PARP targets for cancer therapy. Nat Rev Cancer 14: 502-509, 2014.

23. Gonzalez-Flores A, Aguilar-Quesada R, Siles E, et al: Interaction between PARP-1 and HIF-2 $\alpha$ in the hypoxic response. Oncogene 33: 891-898, 2014

24. Pan R, Chen C, Liu WL and Liu KJ: Zinc Promotes the Death of Hypoxic Astrocytes by Upregulating HypoxiaDInduced Hypoxia-Inducible Factor-1alpha Expression via Poly (ADP-ribose) Polymerase-1. CNS Neurosci Ther 19: 511-520, 2013.

25. Erdélyi K, Pacher P, Virág L and Szabó C: Role of poly(ADP-ribosyl) ation in a 'two-hit' model of hypoxia and oxidative stress in human A549 epithelial cells in vitro. Int J Mol Med 32: 339-346, 2013.

26. Therasse P, Arbuck SG, Eisenhauer EA, Wanders J, Kaplan RS, Rubinstein L, Verweij J, Van Glabbeke M, van Oosterom AT, Christian MC and Gwyther SG: New guidelines to evaluate the response to treatment in solid tumors. European Organization for research and treatment of cancer, national cancer institute of the United States, national cancer institute of Canada. J Natl Cancer Inst 92: 205-216, 2000.

27. Geng R, Chen Z, Zhao X, Qiu L, Liu X, Liu R, Guo W, He G, Li J and Zhu X: Oxidative stress-related genetic polymorphisms are associated with the prognosis of metastatic gastric cancer patients treated with epirubicin, oxaliplatin and 5-fluorouracil combination chemotherapy. PLoS One 9: e116027, 2014.

28. Shi YY and He L: SHEsis, a powerful software platform for analyses of linkage disequilibrium, haplotype construction, and genetic association at polymorphism loci. Cell Res 15: 97-98, 2005.

29. Tregouet DA and Garelle V: A new JAVA interface implementation of THESIAS: Testing haplotype effects in association studies. Bioinformatics 23: 1038-1039, 2007.

30. Liu X, Chen Z, Zhao X, Huang M, Wang C, Peng W, Yin J, Li J, He G, Li X and Zhu X: Effects of IGF2BP2, KCNQ1 and GCKR polymorphisms on clinical outcome in metastatic gastric cancer treated with EOF regimen. Pharmacogenomics 16: 959-970, 2015.

31. Oken MM, Creech RH, Tormey DC, Horton J, Davis TE, McFadden ET and Carbone PP: Toxicity and response criteria of the Eastern Cooperative Oncology Group. Am J Clin Oncol 5: 649-656, 1982

32. Teicher BA: Hypoxia and drug resistance. Cancer Metastasis Rev 13: 139-168, 1994.

33. Galluzzo M, Pennacchietti S, Rosano S, Comoglio PM and Michieli P: Prevention of hypoxia by myoglobin expression in human tumor cells promotes differentiation and inhibits metastasis. J Clin Invest 119: 865-875, 2009.

34. Lu Y, Wajapeyee N, Turker MS and Glazer PM: Silencing of the DNA mismatch repair gene MLH1 induced by hypoxic stress in a pathway dependent on the histone demethylase LSD1. Cell Rep 8: 501-513, 2014.

35. Scanlon SE and Glazer PM: Multifaceted control of DNA repair pathways by the hypoxic tumor microenvironment. DNA Repair (Amst) 32: 180-189, 2015

36. Teng Y, Dai D, Shen W and Liu H: Effect of methylation of hMLH1 gene promotor on stage tumorigenesis and progression of human gastric cancer. Zhonghua Wei Chang Wai Ke Za Zhi 18: 166-170, 2015 (In Chinese).

37. Guo H, Yan W, Yang Y and Guo M: Promoter region methylation of DNA damage repair genes in human gastric cancer. Zhonghua Yi Xue Za Zhi 94: 2193-2196, 2014 (In Chinese). 
38. Li Y, Yang Y, Lu Y, Herman JG, Brock MV, Zhao P and Guo M: Predictive value of CHFR and MLH1 methylation in human gastric cancer. Gastric Cancer 18: 280-287, 2015.

39. Langeberg WJ, Kwon EM, Koopmeiners JS, Ostrander EA and Stanford JL: Population-based study of the association of variants in mismatch repair genes with prostate cancer risk and outcomes. Cancer Epidemiol Biomarkers Prev 19: 258-264, 2010.

40. Chen H, Taylor NP, Sotamaa KM, Mutch DG, Powell MA, Schmidt AP, Feng S, Hampel HL, de la Chapelle A and Goodfellow PJ: Evidence for heritable predisposition to epigenetic silencing of MLH1. Int J Cancer 120: 1684-1688, 2007.

41. Samowitz WS, Curtin K, Wolff RK, Albertsen H, Sweeney C, Caan BJ, Ulrich CM, Potter JD and Slattery ML: The MLH1-93 $\mathrm{G}>\mathrm{A}$ promoter polymorphism and genetic and epigenetic alterations in colon cancer. Genes Chromosomes Cancer 47: 835-844, 2008.
42. Raptis S, Mrkonjic M, Green RC, Pethe VV, Monga N, Chan YM, Daftary D, Dicks E, Younghusband BH, Parfrey PS, et al: MLH1-93G $>$ A promoter polymorphism and the risk of microsatellite-unstable colorectal cancer. J Natl Cancer Inst 99: 463-474, 2007.

43. Zhu H, Li X, Zhang X, et al: Polymorphisms in mismatch repair genes are associated with risk and microsatellite instability of gastric cancer, and interact with life exposures. Gene 579: 52-57, 2016.

44. Choi YY, Bae JM, An JY, Kwon IG, Cho I, Shin HB, Eiji T, Aburahmah M, Kim HI, Cheong JH, et al.: Is microsatellite instability a prognostic marker in gastric cancer? A systematic review with meta-analysis. J Surg Oncol 110: 129-135, 2014.

45. Polom K, Marrelli D, Pascale V, Roviello G, Voglino C, Rho H, Vindigni C, Marini M, Macchiarelli R and Roviello F: High-risk and low-risk gastric cancer areas in Italy and its association with microsatellite instability. J Cancer Res Clin Oncol 142: 18171824, 2016. 